Arq. Bras. Med. Vet. Zootec., v.68, n.2, p.483-488, 2016

\title{
Efeitos da granulometria e da forma física da ração sobre o desempenho de frangos de corte
}

\author{
[Effects of particle size and physical form of diets on broiler performance] \\ E.C. Andrade, N.C. Baião, L.J.C. Lara, J.S.R. Rocha, G. Brumano, \\ M.M. Saldanha, A.R.C. Abreu \\ Escola de Veterinária - Universidade Federal de Minas Gerais - Belo Horizonte, MG \\ RESUMO
}

Foram utilizados 936 pintos de corte machos e 1008 fêmeas da linhagem Cobb® criados no período de um a 45 dias. Os tratamentos foram compostos por duas formas físicas da ração (farelada e peletizada) e duas granulometrias do milho $(3,8 \mathrm{~mm}$ e $7,0 \mathrm{~mm})$. O delineamento experimental foi inteiramente ao acaso, em arranjo fatorial 2 × 2 (duas granulometrias x duas formas físicas), com seis repetições de 39 aves cada para os machos e seis repetições de 42 aves cada para as fêmeas. A ração peletizada produzida com moagem 3,8mm apresentou melhor PDI $(P \leq 0,05)$. Aves fêmeas alimentadas com ração peletizada apresentaram maior consumo de ração, ganho de peso e melhor conversão alimentar que aquelas que receberam ração farelada. Não foi observada diferença significativa $(\mathrm{P}>0,05)$ entre os tratamentos para as variáveis ganho de peso, conversão alimentar e consumo de ração dos machos. Não houve influência da forma física e da granulometria sobre a viabilidade das aves em nenhuma fase de criação $(\mathrm{P}>0,05)$.

Palavras-chave: desempenho, frango de corte, nutrição, pélete

\begin{abstract}
A total of 936 male chickens and 1008 female Cobb ${ }^{\circledR}$ were used from 1 to 45 days old. Treatments consisted of two physical forms (mash and pelleted diets) and two particle sizes (corn ground through $3.8 \mathrm{~mm}$ and $7.0 \mathrm{~mm}$ sieves). The experimental design was completely randomized in a $2 \times 2$ factorial design (two particle sizes $x$ two physical forms), with six replicates of 39 birds each for males and six replicates of 42 birds each for females. PDI from the pelleted physical form that was produced with $3.8 \mathrm{~mm}$ grinding showed better results $(P \leq 0.05)$. Female birds fed pelleted diets had greater feed intake, weight gain and better feed conversion than those fed mash diet. No significant difference (P>0.05) was observed between the treatments for male feed intake, weight gain and feed conversion from 1 to 45 days of age. There was no influence of the physical form and particle size on the feasibility of birds in any phase $(P>0.05)$.
\end{abstract}

Keywords: performance, broiler chicken, animal nutrition, pellet

\section{INTRODUÇÃO}

A ração peletizada é a mais utilizada para frangos de corte no Brasil (Tavernari et al., 2013) e a peletização é o processo mais utilizado para melhorar a qualidade de rações para frangos de corte entre os processamentos térmicos de ração (Massuquetto, 2014).

Recebido em 5 de junho de 2015

Aceito em 18 de fevereiro de 2016

E-mail: evelinecandrade@gmail.com
Uma abrangente literatura correlaciona o uso da peletização com benefícios na produção de frangos de corte. Porém, para a obtenção de bons resultados de desempenho proporcionados por essa ração, é fundamental que se tenha pélete de boa qualidade. Tavernari et al. (2013) afirmam que o processo de peletização de rações requer um cuidado especial porque, uma vez comprometido, poderá haver consumo excessivo de energia elétrica e resultar em uma ração de qualidade inferior, não obtendo os benefícios esperados. 
A granulometria dos ingredientes está diretamente relacionada à qualidade de pélete. A literatura relata que quanto menor a granulometria dos alimentos, melhor o pélete e maior o custo da moagem da ração. Dessa forma, para reduzir os custos de fabricação da ração, é necessário que seja definida também uma granulometria que permita desempenho adequado das aves, associada à redução no consumo de energia elétrica e ao aumento no rendimento de moagem do ingrediente para que o processo compense financeiramente.

Diante do exposto, os objetivos do presente trabalho foram avaliar a influência da granulometria do milho e da forma física da ração sobre o desempenho de frangos de corte fêmeas e machos, de um a 45 dias de idade.

\section{MATERIAL E MÉTODOS}

Um total de 1944 pintos de corte da linhagem Cobb (sendo 936 machos e 1008 fêmeas) de um dia de idade foi alojado em um galpão convencional, utilizando-se cepilho de madeira como material de cama. As fêmeas foram alojadas em 24 boxes, com a densidade de 14 aves $/ \mathrm{m}^{2}$ (42 aves por boxe), e os machos também foram divididos em outros 24 boxes, porém na densidade de 13 aves $/ \mathrm{m}^{2}$ (39 aves por boxe). Água e ração foram oferecidas à vontade. Durante os primeiros 14 dias de idade, os pintos foram aquecidos com uma lâmpada infravermelha (250 Watts) por boxe.

O programa de luz utilizado foi: de um a 14 dias, 24 horas de luz por dia, e a partir do $15^{\circ}$ dia de idade até o final do experimento o período de luminosidade diária foi de 17 horas (natural e artificial).

Os tratamentos foram definidos pela granulometria do milho e pela forma física das rações, sendo: ração farelada $3,8 \mathrm{~mm}$ (milho moído em peneira $3,8 \mathrm{~mm}$ ), farelada $7,0 \mathrm{~mm}$ (milho moído em peneira 7,0mm), peletizada $3,8 \mathrm{~mm}$ (milho moído em peneira $3,8 \mathrm{~mm}$ ) e peletizada $7,0 \mathrm{~mm}$ (milho moído em peneira 7,0mm).

Os tratamentos foram distribuídos em três fases de criação (inicial, um a 23 dias; crescimento, 24 a 40 dias; e final, 41 a 45 dias de idade). As rações foram isonutritivas, sendo os valores nutricionais dos ingredientes utilizados pela indústria local.

A composição percentual das rações com seus respectivos valores nutricionais calculados é apresentada na Tab. 1.

O moinho utilizado foi de martelo e com moagem conjunta com velocidade de $1720 \mathrm{rpm}$; potência de $250 \mathrm{CV}$ e capacidade de moagem de 50 toneladas por hora. As rações peletizadas iniciais foram trituradas após a peletização.

A peletizadora utilizada foi a Van Aarsen International ${ }^{\circledR} 13 \mathrm{~V}$, tipo $\mathrm{C} 900$, com capacidade de 30-33 toneladas/hora.

Antes do alojamento, todos os pintos correspondentes a cada repetição foram pesados em grupos de 39 para os machos e de 42 para as fêmeas para cálculos do desempenho zootécnico das aves. $O$ cálculo de conversão alimentar foi feito com base no consumo médio de ração e no ganho médio de peso dos frangos ao final da fase de criação.

O delineamento experimental para as análises das rações (DGM e PDI) foi inteiramente ao acaso, em arranjo fatorial 2 x 2 (duas granulometrias $\mathrm{x}$ duas formas físicas), com seis repetições por tratamento, sendo cada repetição constituída de um pool de amostras coletadas em diferentes pontos após o resfriador. Somente o DGM da ração inicial violou os princípios de normalidade e homocedasticidade, o qual foi analisado pelo teste de Mann-Whitney mediante o uso do InStat. As outras variáveis analisadas respeitaram os princípios de normalidade e homocedasticidade e foram submetidas à análise de variância, e as diferenças entre as médias foram analisadas pelos testes F e de Tukey, utilizando-se o SAEG UFV.

A metodologia utilizada neste experimento foi aprovada pelo Comitê de Ética em Experimentação Animal da Universidade Federal de Minas Gerais, sob protocolo de número $323 / 2013$. 
Efeitos da granulometria...

Tabela 1. Composição percentual das rações com os respectivos valores nutricionais calculados, de acordo com a fase de criação de frangos de corte

\begin{tabular}{|c|c|c|c|}
\hline Ingredientes & $\begin{array}{c}\text { Inicial } \\
1 \text { a } 23 \text { dias }\end{array}$ & $\begin{array}{l}\text { Crescimento } \\
24 \text { a } 40 \text { dias }\end{array}$ & $\begin{array}{l}\text { Acabamento } \\
41 \text { a } 45 \text { dias }\end{array}$ \\
\hline Gérmen de milho & - & $1,32 \%$ & $1,50 \%$ \\
\hline Gordura de aves & $1,31 \%$ & $1,30 \%$ & $1,20 \%$ \\
\hline Farinha de carne e osso $40 \%$ & $1,82 \%$ & $1,12 \%$ & $0,85 \%$ \\
\hline Sal comum & $0,34 \%$ & $0,31 \%$ & $0,31 \%$ \\
\hline Calcário 38\% & $0,64 \%$ & $0,69 \%$ & $0,69 \%$ \\
\hline Premix vitamínico & $0,03 \%$ & $0,02 \%$ & $0,02 \%$ \\
\hline Premix mineral & $0,05 \%$ & $0,05 \%$ & $0,03 \%$ \\
\hline Farelo soja & $29,53 \%$ & $21,66 \%$ & $20,28 \%$ \\
\hline Milho & $62,43 \%$ & $66,99 \%$ & $68,72 \%$ \\
\hline Farinha de penas & - & $2,50 \%$ & $2,50 \%$ \\
\hline Farinha de vísceras & $3,00 \%$ & $3,10 \%$ & $3,10 \%$ \\
\hline Fitase & $0,01 \%$ & $0,01 \%$ & $0,01 \%$ \\
\hline DL-Metionina $98 \%$ & $0,28 \%$ & $0,26 \%$ & $0,24 \%$ \\
\hline Lisina (Biolys $\left.{ }^{\circledR}\right)$ & $0,32 \%$ & $0,44 \%$ & $0,43 \%$ \\
\hline Treonina $98 \%$ & $0,12 \%$ & $0,11 \%$ & $0,10 \%$ \\
\hline Colina $60 \%$ & $0,05 \%$ & $0,04 \%$ & $0,02 \%$ \\
\hline Surmax $® 200$ & $0,005 \%$ & $0,005 \%$ & $0,005 \%$ \\
\hline Coxistac $\AA 12 \%$ & - & $0,06 \%$ & - \\
\hline \multicolumn{4}{|l|}{ Valores nutricionais calculados } \\
\hline Proteína bruta \% & 21,5 & 20,21 & 19,57 \\
\hline Cálcio \% & 0,88 & 0,79 & 0,75 \\
\hline Fósforo disponível \% & 0,43 & 0,38 & 0,36 \\
\hline Energia metabolizável kcal/kg & 3.050 & 3.150 & 3.170 \\
\hline Lisina digestível $\%$ & 1,16 & 1,06 & 1,02 \\
\hline Metionina digestível \% & 0,57 & 0,52 & 0,49 \\
\hline Metionina + cistina $\%$ digestível & 0,85 & 0,81 & 0,78 \\
\hline Treonina digestível \% & 0,79 & 0,72 & 0,69 \\
\hline Triptofano digestível \% & 0,21 & 0,18 & 0,17 \\
\hline Sódio \% & 0,19 & 0,18 & 0,18 \\
\hline
\end{tabular}

\section{RESULTADOS E DISCUSSÃO}

$\mathrm{Na}$ Tab. 2, são apresentados os valores obtidos de DGM e PDI, de todas as rações, de acordo com as fases de criação.

Não houve diferença entre os PDIs das rações peletizadas moídas em peneiras $3,8 \mathrm{~mm}$ e $7,0 \mathrm{~mm}$ $(\mathrm{P} \leq 0,05)$ nas fases de um a 23 dias e de 24 a 40 dias, e observou-se melhor qualidade de pélete na ração peletizada $3,8 \mathrm{~mm}$ do que na peletizada $7,0 \mathrm{~mm}$ apenas para a fase de 41 a 45 dias de idade.

Esses resultados podem ter sido decorrentes de alterações durante alguma etapa do processo de fabricação, como a variabilidade dos ingredientes, ou durante a peletização de fato (temperatura, umidade, pressão), que influenciam diretamente a qualidade de pélete como afirmado por Biagi (1998).

A afirmação de que o tamanho da partícula dos grãos pode influenciar a integridade ou a durabilidade dos péletes (Biagi, 1998 e Waldroup, 1997) se aplicou apenas para a ração utilizada na fase de 41 a 45 dias de idade. O PDI dessa ração foi significativamente maior $(\mathrm{P} \leq 0,05)$, demonstrando melhor qualidade de pélete para rações peletizadas moídas na peneira de $3,8 \mathrm{~mm}$ do que na de $7,0 \mathrm{~mm}$. 
Tabela 2. DGM e PDI de acordo com a granulometria para as fases: de um a 23 dias, de 23 a 41 dias e de 41 a 45 dias para frangos de corte

\begin{tabular}{llll}
\hline \multirow{2}{*}{ Granulometria } & \multicolumn{3}{c}{ DGM } \\
\cline { 2 - 4 } & $1 \mathrm{a} 23$ dias $^{* *}$ & $24 \mathrm{a} 40$ dias $^{*}$ & $41 \mathrm{a} 45$ dias $^{*}$ \\
\hline $3,8 \mathrm{~mm}$ & $868,8 \mathrm{~b}$ & $890,5 \mathrm{~b}$ & $904,1 \mathrm{~b}$ \\
$7,0 \mathrm{~mm}$ & $1029,4 \mathrm{a}$ & $1058,1 \mathrm{a}$ & 3,1 \\
CV $(\%)$ & 1,7 & 3,1 & $41 \mathrm{a} 45$ dias $^{*}$ \\
\hline \multirow{2}{*}{ GRANULOMETRIA } & \multicolumn{2}{c}{ PDI } \\
\cline { 2 - 4 } & $1 \mathrm{a} 23$ dias $^{*}$ & $24 \mathrm{a} 40$ dias $^{*}$ & $80,0 \mathrm{a}$ \\
3,8mm & $70,4 \mathrm{a}$ & $77,9 \mathrm{a}$ & $75,1 \mathrm{~b}$ \\
CV $(\%)$ & $76,8 \mathrm{a}$ & $75,9 \mathrm{a}$ & 2,4 \\
\hline
\end{tabular}

Médias seguidas de letras minúsculas distintas na coluna diferem entre si pelo teste $\mathrm{F}^{*}$ e de Mann-Whitney (apenas para DGM de um a 23 dias $)^{* *}(\mathrm{P} \leq 0,05)$.

Independentemente da granulometria, o percentual de péletes íntegros pode ser considerado satisfatório de acordo com McKinney e Teeter (2002). Segundo esses autores, as rações com no mínimo $40 \%$ de péletes íntegros já apresentam os benefícios proporcionados pela peletização. Porém, esse percentual está abaixo do recomendado por
Dozier III (2001), que afirma que o PDI ideal para frangos de corte seria de $80 \%$. Portanto, apenas a ração peletizada fina usada na fase de 41 a 45 dias de idade alcançou esse índice.

O desempenho das fêmeas de um a 45 dias de idade, de acordo com os tratamentos, é apresentado na Tab. 3.

Tabela 3. Desempenho de fêmeas de corte de um a 45 dias de idade de acordo com os tratamentos

\begin{tabular}{|c|c|c|c|c|}
\hline \multirow{2}{*}{ Forma física } & \multicolumn{2}{|c|}{ Moagem } & \multirow{2}{*}{ Média } & \multirow[b]{2}{*}{$\mathrm{CV}(\%)$} \\
\hline & $3,8 \mathrm{~mm}$ & $7,0 \mathrm{~mm}$ & & \\
\hline \multicolumn{5}{|c|}{ Consumo de ração (g) } \\
\hline Peletizada & $4595,7 \mathrm{Aa}$ & $4482,0 \mathrm{Ab}$ & 4538,8 & \multirow{3}{*}{1,9} \\
\hline Farelada & $4445,3 \mathrm{Ba}$ & $4481,2 \mathrm{Aa}$ & 4463,2 & \\
\hline Média & 4520,5 & 4481,6 & & \\
\hline \multicolumn{5}{|c|}{ Ganho de peso(g) } \\
\hline Peletizada & 2553,1 & 2532,4 & $2542,7 \mathrm{a}$ & \multirow{3}{*}{2,1} \\
\hline Farelada & 2460 & 2476 & $2468,0 \mathrm{~B}$ & \\
\hline Média & $2506,6 \mathrm{a}$ & $2504,2 \mathrm{a}$ & & \\
\hline \multicolumn{5}{|c|}{ Conversão alimentar (g:g) } \\
\hline Peletizada & 1,8 & 1,77 & $1,78 \mathrm{a}$ & \multirow{3}{*}{1,3} \\
\hline Farelada & 1,81 & 1,81 & $1,81 \mathrm{~B}$ & \\
\hline Média & $1,80 \mathrm{a}$ & $1,79 \mathrm{a}$ & & \\
\hline \multicolumn{5}{|c|}{ Viabilidade $(\%)^{*}$} \\
\hline Peletizada & 98 & 96 & 97 & \multirow{3}{*}{2,5} \\
\hline Farelada & 98,4 & 99,2 & 98,8 & \\
\hline Média & 98,2 & 97,6 & & \\
\hline
\end{tabular}

Médias seguidas de letras distintas, minúsculas na linha e maiúsculas na coluna, diferem estatisticamente entre si $(\mathrm{P} \leq 0,05)$. *Médias não seguidas de letras são semelhantes entre si pelo teste $\mathrm{F}(\mathrm{P}>0,05)$.

Foi observada interação entre os tratamentos (Tab. 3). Entretanto, as aves que receberam ração peletizada moída em peneira de $3,8 \mathrm{~mm}$ apresentaram melhor consumo de ração $(\mathrm{P} \leq 0,05)$ que aquelas alimentadas com ração farelada moída em peneira de $3,8 \mathrm{~mm}$, bem como aquelas que receberam a peletizada moída em peneira de $7,0 \mathrm{~mm}$, porém não houve diferença significativa $(\mathrm{P}>0,05)$ entre os processamentos para a moagem de $7,0 \mathrm{~mm}$ nem para as moagens quando a ração foi farelada. 
Não houve interação entre o tipo de processamento e o grau de moagem para ganho de peso $(\mathrm{P}>0,05)$. Aves que receberam a ração peletizada tiveram maior ganho de peso que aquelas que foram alimentadas com rações fareladas. Esse resultado pode ser explicado pelo fato de a ração peletizada favorecer o consumo, assim como reduzir a taxa de passagem, aumentando a absorção de nutrientes pelas aves. Observação semelhante foi feita por Lara (2007), cujo trabalho mostrou que aves alimentadas com ração peletizada também apresentaram maior ganho de peso de um a 45 dias de idade do que aquelas que receberam ração farelada.

Para as rações peletizadas, a diferença significativa $(\mathrm{P} \leq 0,05) \quad$ observada entre os resultados de PDI (Tab. 2) indica melhor qualidade de pélete para a ração moída em peneira 3,8mm. Apesar dessa observação, a melhoria não foi suficiente para influenciar no ganho de peso das aves (Tab. 3). Isso pode estar relacionado com as observações feitas por McKinney e Teeter (2002), para os quais rações com no mínimo $40 \%$ de péletes íntegros já apresentam os benefícios proporcionados pela peletização, o que diverge de Cutlip et al. (2008), os quais relataram que mesmo pequenas melhorias na qualidade dos péletes podem influenciar positivamente o desempenho de frangos de corte.

Não houve interação entre o tipo de processamento e o grau de moagem $(\mathrm{P}>0,05)$ para a conversão alimentar (Tab. 3). Aves que consumiram ração na forma física peletizada obtiveram melhor conversão alimentar $(\mathrm{P} \leq 0,05)$ do que aquelas que receberam ração farelada. A conversão alimentar das aves que consumiram as rações de ambas as granulometrias foi semelhante. Esse resultado difere parcialmente de López e Baião (2004) e Miranda et al. (2011), que não observaram efeito da granulometria dos ingredientes e da forma física da ração sobre a conversão alimentar dos frangos de corte de um a 47 e de um a 42 dias de idade, respectivamente.

Para a viabilidade de fêmeas (Tab. 3), os resultados não tiveram influência da forma física nem da moagem, sendo semelhantes entre si $(\mathrm{P}>0,05)$.

Tabela 4. Desempenho de machos de corte de um a 45 dias de idade

\begin{tabular}{|c|c|c|c|c|}
\hline \multirow{2}{*}{ Forma física } & \multicolumn{2}{|c|}{ Moagem } & \multirow{2}{*}{ Média } & \multirow{2}{*}{$\mathrm{CV}(\%)$} \\
\hline & $3,8 \mathrm{~mm}$ & $7,0 \mathrm{~mm}$ & & \\
\hline \multicolumn{5}{|c|}{ Consumo de ração $(\mathrm{g})$} \\
\hline Peletizada & 5143,75 & 5110,91 & 5127,33 & \multirow{3}{*}{2,4} \\
\hline Farelada & 5122,58 & 5113,62 & 5118,1 & \\
\hline Média & 5133,17 & 5112,27 & & \\
\hline \multicolumn{5}{|c|}{ Ganho de peso(g) } \\
\hline Peletizada & 2978,38 & 2976,19 & 2977,29 & \multirow{3}{*}{2,5} \\
\hline Farelada & 2941,98 & 2941,27 & 2941,62 & \\
\hline Média & 2960,18 & 2958,73 & & \\
\hline \multicolumn{5}{|c|}{ Conversão alimentar (g:g) } \\
\hline Peletizada & 1,72735 & 1,71814 & 1,72274 & \multirow{3}{*}{1,9} \\
\hline Farelada & 1,74118 & 1,73851 & 1,73985 & \\
\hline Média & 1,73427 & 1,72832 & & \\
\hline \multicolumn{5}{|c|}{ Viabilidade (\%) } \\
\hline Peletizada & 96,5812 & 95,2992 & 95,9402 & \multirow{3}{*}{3,0} \\
\hline Farelada & 97,8633 & 95,7479 & 96,8056 & \\
\hline Média & 97,2222 & 95,5235 & & \\
\hline
\end{tabular}

Médias não seguidas de letras são semelhantes entre si pelo teste $\mathrm{F}(\mathrm{P}>0,05)$.

Não houve interação entre o tipo de processamento e o grau de moagem para consumo de ração, ganho de peso, conversão alimentar e viabilidade $(\mathrm{P}>0,05)$ nem foi observada diferença significativa $(\mathrm{P}>0,05)$ dessas variáveis entre os tratamentos (Tab. 4).

Diante desses resultados, não foi observada preferência das aves pelo tamanho das partículas 
nem pela forma física da ração, diferindo do observado por Portella et al. (1988), para os quais as aves teriam preferência por partículas grossas em detrimento de partículas finas.

Para os machos, a diferença significativa $(\mathrm{P} \leq 0,05)$ observada entre os resultados de PDI (Tab. 2), indicando uma melhor qualidade de pélete para a ração com granulometria moída em peneira $3,8 \mathrm{~mm}$, também não foi suficiente para influenciar o ganho de peso das aves. Como ocorreu com as fêmeas, isto pode estar relacionado com as observações feitas por McKinney e Teeter (2002).

Esses resultados estão de acordo com López e Baião (2004) e Miranda et al. (2011), que não observaram efeito $(\mathrm{P}>0,05)$ da granulometria dos ingredientes e da forma física da ração sobre a conversão alimentar dos frangos de corte de um a 47 e de um a 42 dias de idade, respectivamente.

\section{CONCLUSÕES}

A forma física peletizada moída em peneira de $3,8 \mathrm{~mm}$ apresenta melhor PDI. Para frangos de corte fêmeas, a rações peletizadas resultaram em maior ganho de peso e melhor conversão alimentar, sem haver influência da granulometria, e as rações peletizadas moídas em peneira de $3,8 \mathrm{~mm}$ proporcionaram maior consumo de ração. Para os machos, a forma física da ração e a moagem não têm influência sobre os resultados de desempenho. A forma física e a granulometria não apresentam efeitos sobre a viabilidade dos frangos de ambos os sexos.

\section{REFERÊNCIAS}

BIAGI, J.D. Implicações da granulometria de ingredientes na qualidade $\mathrm{d}$ e pelets e na economia da produção de rações (Revisão). In: SIMPÓSIO SOBRE GRANULOMETRIA DE INGREDIE NTES E RAÇÕES PARA SUÍNOS E AVES, 1998, Concórdia, SC. Anais... Concórdia, SC : EMBRAPA/CNPSA, 1998. p.57.
CUTLIP, S.E., HOTT, J.M., BUCHANAN, N.P. et al., The effect of steam-conditioning practices on pellet quality and growing broiler nutritional value. $J$. Appl. Poulry Res., v.17, p.249-261, 2008.

DOZIER III, W.A. Cost-effective pellet quality for meat birds. Feed Manag. v.52, p.3, 2001.

LARA, L.J.C. Efeitos do processamento da ração e da linhagem sobre os valores energéticos e desempenho de frangos de corte, 2007. 52p.f. Tese (Doutorado em Zootecnia) - Escola de Veterinária, Universidade Federal de Minas Gerais, Belo Horizonte, MG.

LOPEZ, C.A.A, BAIAO, N.C. Efeitos do tamanho da partícula e da forma física da ração sobre o desempenho, rendimento de carcaça e peso dos órgãos digestivos de frangos de corte. Arq. Bras. Med. Vet. Zootec., v.56, p.214-221, 2004.

MASSUQUETTO A. Avaliação da forma física da dieta e do tempo de condicionamento no processo de peletização de dietas para frangos de corte. 2014. 71f. Dissertação (Mestrado em Ciências Veterinárias) - Universidade Federal do Paraná, Curitiba, PR.

McKINNEY, L.; TEETER, R. Caloric value of pelleting. Tech. Focus Cobb, n.3, p.1-6, 2002.

MIRANDA, D. J. A.; LARA, L. J. C.; BAIAO, N. C. et al. Efeito da granulometria e do nível de energia sobre o desempenho de frangos de corte de 23 a 42 dias de idade. In: CONGRESSO LATINOAMERICANO DE AVICULTURA, 22.. 2011, Buenos Aires. Anais... Buenos Aires: ALA, 2011. p.19-56.

PORTELLA, F.J.; CASTON, L.J.; LEESON, S. Apparent feed particle size preference by laying hens. Can. J. Anim. Sci., v.68, p. 915-922, 1988.

TAVERNARI, F.C., LIMA, G.J.M.M., MANZKE, N.E. et al. Resultados da Embrapa com peletização e uso de glicerina bruta. Avic. Ind., n.10, p.14-19, 2013.

WALDROUP, P.W. Particle size reduction of cereal grains and its significance in poultry nutrition. Singapore: American Soybean Association, 1997. 14p. (ASA Technical Bulletin, PO34).

ZANOTTO D.L; BELLAVER C. Método de determinação da granulometria de ingredientes para uso de rações de suínos e aves. Concórdia: EMBRAPA / CNPSA, 1996. 